\title{
Prevalence of pathogenic BRCA1/2 germline mutations among 802 women with unilateral triple-negative breast cancer without family cancer history
}

Christoph Engel ${ }^{1 \dagger}$, Kerstin Rhiem ${ }^{2 \dagger}$, Eric Hahnen ${ }^{2}$, Sibylle Loibl ${ }^{3,4}$, Karsten E. Weber ${ }^{3}$, Sabine Seiler ${ }^{3}$, Silke Zachariae ${ }^{1}$, Jan Hauke ${ }^{2}$, Barbara Wappenschmidt ${ }^{2}$, Anke Waha², Britta Blümcke², Marion Kiechle, ${ }^{5}$ Alfons Meindl ${ }^{5}$, Dieter Niederacher ${ }^{6}$, Claus R. Bartram ${ }^{7}$, Dorothee Speiser ${ }^{8}$, Brigitte Schlegelberger ${ }^{9}$, Norbert Arnold ${ }^{10}$, Peter Wieacker ${ }^{11}$, Elena Leinert ${ }^{12}$, Andrea Gehrig ${ }^{13}$, Susanne Briest ${ }^{14}$, Karin Kast ${ }^{15,16,17}$, Olaf Riess ${ }^{18}$, Günter Emons ${ }^{19}$, Bernhard H. F. Weber ${ }^{20}$, Jutta Engel ${ }^{21}$, Rita K. Schmutzler ${ }^{2 *}$ and on behalf of the German Consortium for Hereditary Breast and Ovarian Cancer (GC-HBOC)

\begin{abstract}
Background: There is no international consensus up to which age women with a diagnosis of triple-negative breast cancer (TNBC) and no family history of breast or ovarian cancer should be offered genetic testing for germline BRCA1 and BRCA2 (gBRCA) mutations. Here, we explored the association of age at TNBC diagnosis with the prevalence of pathogenic gBRCA mutations in this patient group.

Methods: The study comprised 802 women (median age 40 years, range 19-76) with oestrogen receptor, progesterone receptor, and human epidermal growth factor receptor type 2 negative breast cancers, who had no relatives with breast or ovarian cancer. All women were tested for pathogenic gBRCA mutations. Logistic regression analysis was used to explore the association between age at TNBC diagnosis and the presence of a pathogenic gBRCA mutation.

Results: A total of 127 women with TNBC (15.8\%) were gBRCA mutation carriers (BRCA1: $n=118,14.7 \% ; B R C A 2: n=9,1$. 1\%). The mutation prevalence was $32.9 \%$ in the age group 20-29 years compared to 6.9\% in the age group 60-69 years. Logistic regression analysis revealed a significant increase of mutation frequency with decreasing age at diagnosis (odds ratio 1.87 per 10 year decrease, $95 \% \mathrm{Cl} 1.50-2.32, p<0.001$ ). gBRCA mutation risk was predicted to be $>10 \%$ for women diagnosed below approximately 50 years.

Conclusions: Based on the general understanding that a heterozygous mutation probability of $10 \%$ or greater justifies gBRCA mutation screening, women with TNBC diagnosed before the age of 50 years and no familial history of breast and ovarian cancer should be tested for gBRCA mutations. In Germany, this would concern approximately 880 women with newly diagnosed TNBC per year, of whom approximately 150 are expected to be identified as carriers of a pathogenic gBRCA mutation.
\end{abstract}

Keywords: Hereditary breast and ovarian cancer, BRCA1, BRCA2, Triple-negative breast cancer

\footnotetext{
* Correspondence: rita.schmutzler@uk-koeln.de

${ }^{\dagger}$ Equal contributors

${ }^{2}$ Center for Hereditary Breast and Ovarian Cancer and Center for Integrated

Oncology (ClO), Medical Faculty, University Hospital Cologne, Kerpener

Strasse 34, 50931 Cologne, Germany

Full list of author information is available at the end of the article
} 


\section{Background}

Triple-negative breast cancer (TNBC) is characterized by lacking expression of oestrogen receptor (ER), progesterone receptor (PR), and human epidermal growth factor receptor type 2 (HER2). TNBC has been reported to account for $12-24 \%$ of all breast cancers and is associated with a hereditary disease cause [1-3]. While germline $B R C A 1 / 2$ (gBRCA) mutations are found in about $5 \%$ of all breast cancers, higher mutation rates are observed in TNBC patients depending on age of onset and the presence of a family history of breast and ovarian cancer [4]. Approximately $70 \%$ of breast cancers arising in BRCA1 mutation carriers and up to $23 \%$ of breast cancers in BRCA2 carriers are triple-negative [2]. Consequently, some guidelines suggest genetic gBRCA mutation testing in this particular group of patients. For example, the National Institute for Health and Care Excellence (NICE) guidelines recommend gBRCA testing if the combined BRCA1 and BRCA2 mutation carrier probability is expected to be at least 10\% [5]. According to the National Comprehensive Cancer Network (NCCN) and the American Society of Breast Surgeons, gBRCA testing is generally advised for women with TNBC diagnosed at an age of $\leq 60$ years, irrespective of a positive cancer family history.

It is less clear, however, up to which age at TNBC diagnosis women, who do not have a family history of breast and ovarian cancer, should be recommended genetic testing. This prompted us to determine the agedependent prevalence of gBRCA mutations in women with unilateral TNBC and without any family history of breast or ovarian cancer.

\section{Methods}

\section{Study population}

The study population comprised a total of 802 women with TNBC, who reported not to have any relatives with breast or ovarian cancer in their families. Of these, 649 women were consecutively registered and documented between July 1999 and January 2016 in 15 specialized university centres of the German Consortium for Hereditary Breast and Ovarian Cancer (GC-HBOC). GC-HBOC collects data on families suspected of having hereditary breast and ovarian cancer based on a defined set of clinical ascertainment criteria [6]. At the time of data analysis, a total of 2029 women with TNBC were recorded in the GC-HBOC registry (ie., 1380 with and 649 without a family history of breast and ovarian cancer). Women with a TNBC diagnosis before age 36 , who did not have any familial cancer history, were included since 1999, while women with a later TNBC diagnosis were collected mainly since 2011, resulting in some overrepresentation of women with younger age at TNBC diagnosis. Another 153 women were taken from the randomized controlled GeparSixto (GBG 66, clinicaltrials.gov identifier: NCT01426880) trial of the German Breast Group (GBG) [7, 8]. These women were consecutively recruited between August 2011 and December 2012. Women with syn- or metachronous bilateral breast cancer and/or ovarian cancer were excluded. Family history was collected and documented by a medical health professional (geneticist, genetic counselor, and/or gynecologist) after the diagnosis of TNBC. Pedigree information was required to encompass at least three generations.

The multicentre GC-HBOC registry and the multicentre GBG GeparSixto trial have been approved by the responsible ethics committees. Written informed consent to be enrolled in the GC-HBOC registry or the GBG GeparSixto trial, respectively, was obtained from all individuals whose data was used for the present analysis.

\section{Receptor status and mutation analysis}

ER, PR and HER2 expression was determined according to the national pathology guidelines of the 'AGO Breast Committee', which adheres closely to international standards. Triple negativity was defined as immunohistochemical staining of less than $1 \%$ of nuclei for both ER and PR, and an immunhistochemical result (DAKO score) of 0 or $1+$ for Her2/neu. Mutation analyses were performed using either next generation sequencing methods or denaturing high-performance liquid chromatography and high-resolution melting followed by direct Sangerbased sequencing of conspicuous fragments [9, 10]. If no deleterious sequence alterations were found in these analyses, samples were screened for large genomic alterations in the $B R C A 1 / 2$ genes by Multiplex Ligation-dependent Probe Amplification (MLPA) with the $\mathrm{SALSA}^{\oplus} \mathrm{MLPA}^{\oplus}$ probemixes P002 for BRCA1 and P045 for BRCA2 (MRCHolland, Amsterdam, The Netherlands) according to the manufacturer's protocol. Mutations were classified according to the International Agency for Research on Cancer (IARC) system and considered pathogenic or likely pathogenic (class 4 or 5) based on literature evidence, multifactorial likelihood and functional analyses of the ENIGMA consortium that comprises genetic data of the GC-HBOC database $[11,12]$.

\section{Statistical analysis}

Logistic regression was used to analyse the association between age of diagnosis and the presence of a gBRCA mutation. Ninety-five percent confidence intervals for frequencies were calculated applying Wilson's score method. For the calculation of the expected annual number of gBRCA mutation carriers among women with TNBC and without any relatives with breast or ovarian cancer, data on the age-specific annual numbers of all breast cancers in Germany were obtained from pooled data of the German Epidemiological Cancer Registries (German Centre for Cancer Registry Data at the Robert 
Table 1 Basic patient characteristics

\begin{tabular}{llll}
\hline & Total $n=802$ & GC-HBOC $n=649$ & GBG $n=153$ \\
\hline BRCA mutation status, no (\%) & & & $138(90.2)$ \\
negative & $675(84.2)$ & $1037(82.7)$ & $11(7.2)$ \\
BRCA1 & $118(14.7)$ & $5(0.8)$ & $4(2.6)$ \\
BRCA2 & $9(1.1)$ & $38.0(19.5-76.2)$ & $48.0(21.0-74.0)$ \\
Age at diagnosis, years (median, range) & $40.1(19.5-76.2)$ & $38.7(19.5-76.2)$ & $48.5(26.0-74.0)$ \\
negative & $42.0(19.5-76.2)$ & $33.5(25.0-63.5)$ & $41.0(21.0-61.0)$ \\
BRCA1 & $34.1(21.0-63.5)$ & $47.1(34.3-63.1)$ & $54.0(39.0-63.0)$ \\
BRCA2 & $47.2(34.3-63.1)$ & &
\end{tabular}

Koch-Institute, Berlin, Germany). Age-specific frequencies of TNBC cases among all breast cancers were kindly provided by the Munich Cancer Registry (MCR, Munich, Germany). The age-specific proportions of TNBC without a positive family history among unselected TNBC were taken from Couch et al. [13]. P-values $<0.05$ were considered significant. Statistical analyses were conducted using IBM SPSS Statistics for Windows Version 23.0 (IBM Corporation, Armonk, NY, USA) and R 3.3.2 for Windows (R Core Team, www.r-project.org).

\section{Results}

A total of 802 patients were included in the study (649 from the GC-HBOC registry and 153 from the GBG randomized controlled GeparSixto trial). Basic patient characteristics are shown in Table 1 . The median age at TNBC diagnosis was 40 (range 19-76) years and the overall prevalence of pathogenic gBRCA mutations was $15.8 \%$ (14.7\% for BRCA1 and $1.1 \%$ for BRCA2). BRCA1 mutation carriers were younger at diagnosis (34 years) than BRCA2 mutation carriers (47 years). Non-carriers had a median age at diagnosis of 42 years. Patients from the GeparSixto study were older at primary TNBC diagnosis compared to the patients from the GC-HBOC registry (48 vs. 38 years), and had a lower mutation prevalence (9.8\% vs. $17.3 \%)$. The considerably lower age at $\mathrm{TNBC}$ diagnosis of $\mathrm{GC}-\mathrm{HBOC}$ patients is explained by an overrepresentation of women diagnosed before the age of 36 years (see Materials). Table 2 depicts the agegroup specific $B R C A 1$ and $B R C A 2$ mutation prevalence. The mutation prevalence increased with younger age at diagnosis. While in about one third of the very young patients (20 to 29 years) a gBRCA mutation was detected (33\%), the prevalence was below $7 \%$ in older patients between 60 and 69 years.

To assess the association between age of TNBC at diagnosis and gBRCA mutation prevalence, a logistic regression analysis was performed (Fig. 1). This analysis revealed a significant negative association between age at diagnosis and the presence of a gBRCA mutation (OR 1.87 per 10 year decrease, $95 \%$ CI 1.50 to $2.32, p<0.001$ ). At an age of approximately 48 years or younger, the predicted mutation probability was $>10 \%$, which is the currently accepted international threshold to recommend gBRCA testing according to the NICE guidelines [5]. The origin of the data (GC-HBOC registry versus GBG GeparSixto trial) did not play a role as a significant confounder in the regression analysis $(p=0.823)$.

The performance of age at TNBC diagnosis to discriminate between gBRCA carriers and non-carriers, as characterized by the area under the curve of the receiver-operating-characteristic (ROC-AUC), was 67.0\% (95\%CI 62.1-71.9\%). Recommendation of genetic testing only to TNBC patients diagnosed before the age of

Table 2 BRCA 1/2 germline mutation prevalence by age group

\begin{tabular}{|c|c|c|c|c|c|c|c|}
\hline & \multirow[t]{2}{*}{$n$} & \multicolumn{2}{|c|}{$B R C A 1$} & \multicolumn{2}{|c|}{$B R C A 2$} & \multicolumn{2}{|c|}{ BRCA1/2 } \\
\hline & & $\bar{n}$ & $\%(95 \% \mathrm{Cl})$ & $\bar{n}$ & $\%(95 \% \mathrm{Cl})$ & $\bar{n}$ & $\%(95 \% \mathrm{Cl})$ \\
\hline \multicolumn{8}{|c|}{ Age group (years) } \\
\hline $20-29$ & 85 & 28 & 32.9 (23.9-43.5) & 0 & $0.0(0.0-4.3)$ & 28 & $32.9(23.9-43.5)$ \\
\hline $30-39$ & 309 & 60 & $19.4(15.4-24.2)$ & 3 & $1.0(0.3-2.8)$ & 63 & $20.4(16.3-25.2)$ \\
\hline $40-49$ & 216 & 22 & $10.2(6.8-14.9)$ & 3 & $1.4(0.5-4.0)$ & 25 & $11.6(8.0-16.5)$ \\
\hline $50-59$ & 122 & 6 & $4.9(2.3-10.3)$ & 1 & $0.8(0.1-4.5)$ & 7 & 5.7 (2.8-11.4) \\
\hline $60-69$ & 58 & 2 & $3.4(1.0-11.7)$ & 2 & $3.4(1.0-11.7)$ & 4 & $6.9(2.7-16.4)$ \\
\hline 70-79 & 12 & 0 & $0.0(0.0-24.2)$ & 0 & $0.0(0.0-24.2)$ & 0 & $0.0(0.0-24.2)$ \\
\hline TOTAL & 802 & 118 & 14.7 (12.4-17.3) & 9 & $1.1(0.6-2.1)$ & 127 & $15.8(13.5-18.5)$ \\
\hline
\end{tabular}




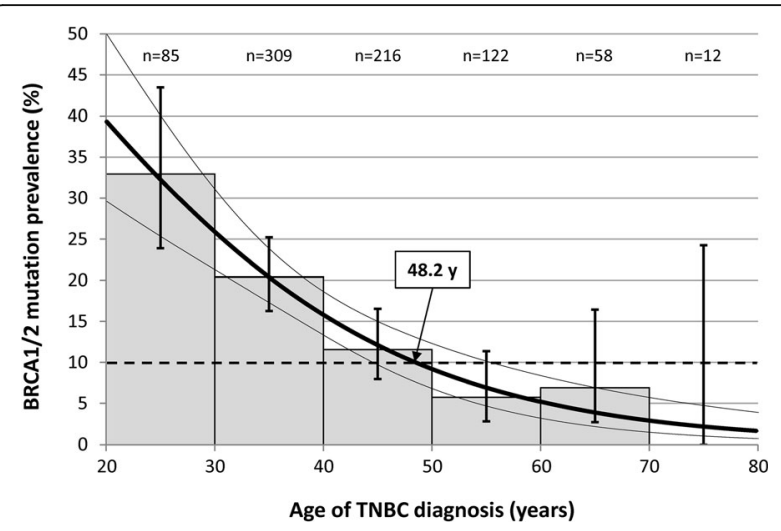

Fig. 1 Association between age of TNBC diagnosis and gBRCA mutation prevalence. Grey shaded bars indicate the mutation prevalence in the according age group (error bars indicate 95\% confidence intervals). The bold curve represents the mutation probability predicted by the logistic regression model (with 95\% confidence band, thin curves). The dashed horizontal line depicts the $10 \%$ mutation probability, above which gBRCA mutation analysis is recommended

50 years would result in a sensitivity of $96.9 \%(95 \% \mathrm{CI}$ 92.2-98.8\%) and a specificity of 9.8\% (95\%CI 7.8-12.3\%).

Table 3 shows an estimation of the expected annual number of TNBC cases with a gBRCA mutation in the general German population, based on the agespecific absolute numbers of all breast cancers up to an age of 80 years and the expected rate of TNBC cases without family history. According to this calculation, the expected frequency of TNBC cases without family history among all breast cancers diagnosed below the age of 50 years would be $26.1 \%$ (882 among 3376 cases), with an expected number of 149 pathogenic gBRCA mutation carriers (positive predictive value of $16.9 \%$ among TNBC cases under 50 years). The expected number of gBRCA carriers among 2494 women with TNBC diagnosed at age 50 or above would amount to 109, corresponding to a negative predictive value of $95.6 \%$.

\section{Discussion}

We conducted the present study to determine up to which age of TNBC diagnosis women without a family history of breast and ovarian cancer should undergo gBRCA testing if an expected mutation probability of at least $10 \%$ is used as decision threshold. This cut-off is currently used as general decision threshold to consider gBRCA testing in Germany.

Our analysis of 802 women revealed that this age cutoff lies at approximately 50 years. However, our analysis also showed that the discriminative performance of age at diagnosis as an exclusive predictor for the presence of a gBRCA mutation was comparatively low with an ROC-AUC of only $67 \%$. If an age cut-off of 50 years would be chosen, the sensitivity would be $97 \%$, with a specificity of $10 \%$. If nationwide gBRCA mutation testing in Germany would be offered to women with newly diagnosed TNBC before the age of 50 years, we expect to identify around 150 additional mutation carriers among around 880 women to be tested per year, corresponding to a positive predictive value of approximately $17 \%$. Among the larger number of approximately 2500 women diagnosed with TNBC at 50 years or later, a total of about 110 mutation carrying women would not be detected (4.4\%; negative predictive value $96.6 \%$ ), which is about $42 \%$ of all expected mutation carriers among TNBC cases up to the age of 80 years.

A number of earlier studies have investigated the gBRCA mutation prevalence in women with TNBC [13-22]. One study based on 207 TNBC cases unselected for family history has also employed logistic regression analysis to describe the association between age at diagnosis and mutation probability accounting for the presence or absence of family history [19]. In this study, the observed mutation prevalence was $6.3 \%(95 \% \mathrm{CI} 1-12 \%)$, which is lower than found in our analysis. Couch et al. presented data on 1508 TNBC cases unselected for family history, for whom information on age at diagnosis and family history was complete [13]. In the subgroup of women with TNBC up to the age

Table 3 Expected annual number of women with TNBC and gBRCA mutation in Germany

\begin{tabular}{lllllll}
\hline Age group & $\begin{array}{l}\text { Number of newly } \\
\text { diagnosed BC } \\
\text { cases in 2012 }\end{array}$ & $\begin{array}{l}\text { \% TNBC among } \\
\text { all BC cases }{ }^{b}\end{array}$ & $\begin{array}{l}\text { \% of TNBC } \\
\text { W/o FH }{ }^{c}\end{array}$ & $\begin{array}{l}\text { Number of TNBC } \\
\text { cases w/o FH }\end{array}$ & $\begin{array}{l}\text { \% BRCA1/2 mutation } \\
\text { carriers among TNBC } \\
\text { cases w/o FH }{ }^{\text {d }}\end{array}$ & $\begin{array}{l}\text { Number of BRCA1/2 } \\
\text { mutation carriers among } \\
\text { TNBC cases without FH }\end{array}$ \\
\hline$<40$ & 2462 & 19.8 & 62.2 & 302 & 26.3 & 79 \\
$40-49$ & 9757 & 10.5 & 56.8 & 580 & 12.1 & 70 \\
$50-59$ & 15,059 & 9.0 & 65.8 & 891 & 6.9 & 61 \\
$60-69$ & 16,497 & 7.0 & 71.9 & 835 & 3.8 & 32 \\
$70-79$ & 15,420 & 6.9 & 71.9 & 768 & 2.1 & 16 \\
\hline
\end{tabular}

FH family history, $B C$ breast cancer, $T N B C$ triple-negative breast cancer

a German Centre for Cancer Registry Data at the Robert Koch-Institute, Berlin, Germany

${ }^{\mathrm{b}}$ Munich Cancer Registry, MCR, Munich, Germany

${ }^{c}$ Couch et al. [13]

dpresent study 
of 59 years without family history they reported a mutation prevalence of $11.2 \%$ (BRCA1 8.1\% and BRCA2 3.0\%).

Current guidelines regarding the age up to which TNBC patients should be offered gBRCA testing vary between countries. The NICE guideline recommends gBRCA mutation testing for women with TNBC and no family history diagnosed before 40 years of age, while the NCCN guideline recommends testing up to the age of 60 years. The normative definition of such decision thresholds depends on the expected mutation probabilities above which insurance carriers are willing to cover the costs for genetic testing. However, the definition of optimal BRCA genetic testing programs and decision cut-offs requires a thorough economic evaluation regarding their cost-effectiveness [23]. Such evaluations are currently not available for Germany but urgently needed.

A limitation of our study is the comparatively low sample size for women with an age at TNBC diagnosis of $\geq 60$ years, resulting in large confidence intervals of the mutation prevalence estimates.

\section{Conclusions}

Based on the understanding that a heterozygous mutation probability of $\geq 10 \%$ justifies gBRCA mutation screening, women with triple-negative breast cancer diagnosed before the age of 50 years and no familial history of breast and ovarian cancer should be tested for gBRCA mutations. In Germany, this would concern approximately 880 women with newly diagnosed TNBC per year, of whom approximately 150 are expected to be identified as pathogenic gBRCA mutation carriers.

\section{Abbreviations \\ BRCA1/2: Breast cancer gene 1 and 2; Cl: Confidence interval; ER: Estrogen receptor; GBG: German Breast Group; gBRCA: germline BRCA1/2 mutation; GC-HBOC: German Consortium for Hereditary Breast and Ovarian Cancer; HER2: Human epidermal growth factor receptor 2; OR: Odds ratio; PR: Progesterone receptor; ROC-AUC: Area under the curve of the receiver operating-characteristic; TNBC: Triple negative breast cancer}

\section{Acknowledgements}

We are indebted to the patients who contributed to this study.

\section{Funding}

The German Consortium for Hereditary Breast and Ovarian Cancer is funded by the German Cancer Aid (grant number 110837). The funding body had no role in the design of the study and collection, analysis, and interpretation of data and in writing the manuscript.

\section{Availability of data and materials}

The datasets used and/or analysed during the current study are available from the corresponding author on reasonable request.

\section{Authors' contributions}

Conception and design, data analysis and interpretation, drafting the manuscript: CE, KR, EH, RKS. Acquisition of data, final approval of the version to be published and agreement to be accountable for all aspects of the work in ensuring that questions related to the accuracy or integrity of any part of the work are appropriately investigated and resolved: $C E, K R, E H, S L$, KEW, SS, SZ, JH, BW, AW, BB, MK, AM, DN, CRB, DS, BS, NA, PW, EL, AG, SB, KK, $\mathrm{OR}, \mathrm{GE}, \mathrm{BHFW}$, JE, RKS. Revising the manuscript critically for important intellectual content: SL, KEW, SS, SZ, JH, BW, AW, BB, MK, AM, DN, CRB, DS, $B S, N A, P W, E L, A G, S B, K K, O R, G E, B H F W$, JE. All authors read and approved the final manuscript.

Ethics approval and consent to participate

The multicentre GC-HBOC registry and the multicentre GBG GeparSixto trial have been approved by the responsible ethics committees. Written informed consent to be enrolled in the GC-HBOC registry or the GBG GeparSixto trial, respectively, was obtained from all individuals whose data was used for the present analysis.

\section{Consent for publication}

Not applicable.

\section{Competing interests}

Eric Hahnen, Kerstin Rhiem, Brigitte Schlegelberger, Rita Schmutzler: Advisory Board Astra Zeneca. Sibylle Loibl is member of the editorial board (Associate Editor) of BMC Cancer. All other authors declare that they have no competing interests.

\section{Publisher's Note}

Springer Nature remains neutral with regard to jurisdictional claims in published maps and institutional affiliations.

\section{Author details}

${ }^{1}$ Institute for Medical Informatics, Statistics and Epidemiology, University of Leipzig, Leipzig, Germany. ${ }^{2}$ Center for Hereditary Breast and Ovarian Cancer and Center for Integrated Oncology $(\mathrm{ClO})$, Medical Faculty, University Hospital Cologne, Kerpener Strasse 34, 50931 Cologne, Germany. ${ }^{3}$ German Breast Group, Neu-Isenburg, Germany. ${ }^{4}$ Centre for Haematology and Oncology Bethanien, Frankfurt, Germany. ${ }^{5}$ Department of Gynecology and Center for Hereditary Breast and Ovarian Cancer, Klinikum rechts der Isar, Technical University Munich (TUM), Munich, Germany. ${ }^{6}$ Department of Gynecology and Obstetrics, University Hospital of the Heinrich-Heine University, Düsseldorf, Germany. ${ }^{7}$ Institute of Human Genetics, University Hospital, University of Heidelberg, Heidelberg, Germany. ${ }^{8}$ Zentrum für Familiären Brust- und Eierstockkrebs, Klinik für Gynäkologie mit Brustzentrum, Charité - Universitätsmedizin Berlin, Berlin, Germany. ${ }^{9}$ Department of Human Genetics, Hannover Medical School, Hannover, Germany. ${ }^{10}$ Institute of Clinical Molecular Biology/Department of Gynecology and Obstetrics, University Hospital of Schleswig-Holstein, Campus Kiel, Christian-Albrechts University Kiel, Kiel, Germany. ${ }^{11}$ Institute of Human Genetics, University Hospital Münster, Münster, Germany. ${ }^{12}$ Department of Gynecology and Obstetrics, University Hospital UIm, Ulm, Germany. ${ }^{13}$ Institute of Human Genetics, University Würzburg, Würzburg, Germany. ${ }^{14}$ Center for Hereditary Breast and Ovarian Cancer, University Hospital Leipzig, Leipzig, Germany.

${ }^{15}$ Department of Gynecology and Obstetrics, Medical Faculty and University Hospital Carl Gustav Carus, Technische Universität Dresden, Dresden, Germany. ${ }^{16}$ National Center for Tumor Diseases (NCT), Partner Site Dresden, Dresden, Germany. ${ }^{17}$ German Cancer Consortium (DKTK), Dresden, and German Cancer Research Center (DKFZ), Heidelberg, Germany. ${ }^{18}$ Institute of Medical Genetics and Applied Genomics, University of Tübingen, Tübingen, Germany. ${ }^{19}$ Klinik für Gynäkologie und Geburtshilfe, Universitätsmedizin, Göttingen, Germany. ${ }^{20}$ Institute of Human Genetics, University of Regensburg, Regensburg, Germany. ${ }^{21}$ Munich Cancer Registry (MCR) of the Munich Tumour Centre (TZM), Institute for Medical Information Processing, Biometry and Epidemiology (IBE), University Hospital of Munich, Ludwig-Maximilians-University (LMU), Munich, Germany.

Received: 27 April 2017 Accepted: 23 January 2018

Published online: 07 March 2018

References

1. Foulkes WD, Smith IE, Reis-Filho JS. Triple-negative breast cancer. N Engl J Med. 2010;363(20):1938-48.

2. Stevens KN, Vachon CM, Couch FJ. Genetic susceptibility to triple-negative breast cancer. Cancer Res. 2013;73(7):2025-30.

3. Metcalfe K, Lubinski J, Lynch HT, Ghadirian P, Foulkes WD, Kim-Sing C, Neuhausen S, Tung N, Rosen B, Gronwald J, et al. Family history of cancer and cancer risks in women with BRCA1 or BRCA2 mutations. J Natl Cancer Inst. 2010;102(24):1874-8. 
4. Network CGA. Comprehensive molecular portraits of human breast tumours. Nature. 2012;490(7418):61-70.

5. National Collaborating Centre for Cancer (UK): Familial Breast Cancer: Classification and Care of People at Risk of Familial Breast Cancer and Management of Breast Cancer and Related Risks in People with a Family History of Breast Cancer. Cardiff (UK): National Collaborating Centre for Cancer (UK); 2013

6. Kast K, Rhiem K, Wappenschmidt B, Hahnen E, Hauke J, Bluemcke B, Zarghooni V, Herold N, Ditsch N, Kiechle M, et al. Prevalence of BRCA1/2 germline mutations in 21401 families with breast and ovarian cancer. J Med Genet. 2016;53(7):465-71.

7. von Minckwitz G, Schneeweiss A, Loibl S, Salat C, Denkert C, Rezai M, Blohmer JU, Jackisch C, Paepke S, Gerber B, et al. Neoadjuvant carboplatin in patients with triple-negative and HER2-positive early breast cancer (GeparSixto; GBG 66): a randomised phase 2 trial. Lancet Oncol. 2014;15(7): 747-56

8. Hahnen E, Lederer B, Hauke J, Loibl S, Krober S, Schneeweiss A, Denkert C, Fasching PA, Blohmer JU, Jackisch C, et al. Germline mutation status, pathological complete response, and disease-free survival in triple-negative breast cancer: secondary analysis of the GeparSixto randomized clinical trial. JAMA Oncol. 2017;3(10):1378-85.

9. Arnold N, Gross E, Schwarz-Boeger U, Pfisterer J, Jonat W, Kiechle M. A highly sensitive, fast, and economical technique for mutation analysis in hereditary breast and ovarian cancers. Hum Mutat. 1999;14(4):333-9.

10. Gross E, Arnold N, Goette J, Schwarz-Boeger U, Kiechle M. A comparison of BRCA1 mutation analysis by direct sequencing, SSCP and DHPLC. Hum Genet. 1999;105(1-2):72-8.

11. Plon SE, Eccles DM, Easton D, Foulkes WD, Genuardi M, Greenblatt MS Hogervorst FB, Hoogerbrugge N, Spurdle AB, Tavtigian SV. Sequence variant classification and reporting: recommendations for improving the interpretation of cancer susceptibility genetic test results. Hum Mutat. 2008; 29(11):1282-91.

12. Spurdle AB, Healey $S$, Devereau A, Hogervorst FB, Monteiro AN, Nathanson KL, Radice P, Stoppa-Lyonnet D, Tavtigian S, Wappenschmidt B, et al. ENIGMA-evidence-based network for the interpretation of germline mutant alleles: an international initiative to evaluate risk and clinical significance associated with sequence variation in BRCA1 and BRCA2 genes. Hum Mutat. 2012:33(1):2-7.

13. Couch FJ, Hart SN, Sharma P, Toland AE, Wang X, Miron P, Olson JE, Godwin AK, Pankratz VS, Olswold C, et al. Inherited mutations in 17 breast cancer susceptibility genes among a large triple-negative breast cancer cohort unselected for family history of breast cancer. J Clin Oncol. 2015;33(4):304-11.

14. Buys SS, Sandbach JF, Gammon A, Patel G, Kidd J, Brown KL, Sharma L, Saam J, Lancaster J, Daly MB. A study of over 35,000 women with breast cancer tested with a 25-gene panel of hereditary cancer genes. Cancer. 2017;123(10):1721-30.

15. Zhang J, Pei R, Pang Z, Ouyang T, Li J, Wang T, Fan Z, Fan T, Lin B, Xie Y. Prevalence and characterization of BRCA1 and BRCA2 germline mutations in Chinese women with familial breast cancer. Breast Cancer Res Treat. 2012; 132(2):421-8.

16. Gonzalez-Rivera M, Lobo M, Lopez-Tarruella S, Jerez Y, Del Monte-Millan M, Massarrah T, Ramos-Medina R, Ocana I, Picornell A, Garzon SS, et al. Frequency of germline DNA genetic findings in an unselected prospective cohort of triple-negative breast cancer patients participating in a platinumbased neoadjuvant chemotherapy trial. Breast Cancer Res Treat. 2016;156(3): 507-15.

17. Gonzalez-Angulo AM, Timms KM, Liu S, Chen H, Litton JK, Potter J, Lanchbury JS, Stemke-Hale K, Hennessy BT, Arun BK, et al. Incidence and outcome of BRCA mutations in unselected patients with triple receptornegative breast cancer. Clin Cancer Res. 2011;17(5):1082-9.

18. Wong-Brown MW, Meldrum CJ, Carpenter JE, Clarke CL, Narod SA, Jakubowska A, Rudnicka H, Lubinski J, Scott RJ. Prevalence of BRCA1 and BRCA2 germline mutations in patients with triple-negative breast cancer. Breast Cancer Res Treat. 2015;150(1):71-80.

19. Sharma P, Klemp JR, Kimler BF, Mahnken JD, Geier LJ, Khan QJ, Elia M, Connor CS, McGinness MK, Mammen JM, et al. Germline BRCA mutation evaluation in a prospective triple-negative breast cancer registry: implications for hereditary breast and/or ovarian cancer syndrome testing. Breast Cancer Res Treat. 2014;145(3):707-14.
20. Rummel S, Varner E, Shriver CD, Ellsworth RE. Evaluation of BRCA1 mutations in an unselected patient population with triple-negative breast cancer. Breast Cancer Res Treat. 2013;137(1):119-25.

21. Fostira F, Tsitlaidou M, Papadimitriou C, Pertesi M, Timotheadou E, Stavropoulou AV, Glentis S, Bournakis E, Bobos M, Pectasides D, et al. Prevalence of BRCA1 mutations among 403 women with triple-negative breast cancer: implications for genetic screening selection criteria: a Hellenic cooperative oncology group study. Breast Cancer Res Treat. 2012;134(1):353-62.

22. Hartman AR, Kaldate RR, Sailer LM, Painter L, Grier CE, Endsley RR, Griffin M, Hamilton SA, Frye CA, Silberman MA, et al. Prevalence of BRCA mutations in an unselected population of triple-negative breast cancer. Cancer. 2012; 118(11):2787-95

23. D'Andrea E, Marzuillo C, De Vito C, Di Marco M, Pitini E, Vacchio MR, Villari P. Which BRCA genetic testing programs are ready for implementation in health care? A systematic review of economic evaluations. Genet Med. 2016;18(12):1171-80.

\section{Submit your next manuscript to BioMed Central and we will help you at every step:}

- We accept pre-submission inquiries

- Our selector tool helps you to find the most relevant journal

- We provide round the clock customer support

- Convenient online submission

- Thorough peer review

- Inclusion in PubMed and all major indexing services

- Maximum visibility for your research

Submit your manuscript at www.biomedcentral.com/submit 